\title{
Humor Style and Creativity Tendency of Senior High School Students of Tujia Ethnic Group in China
}

\author{
Huiyong Fan ${ }^{*}$, Ying Ge $\mathrm{Ge}^{2,3 * \#, ~ R o s s ~ W i l k i n s o n}{ }^{3 \#}$ \\ ${ }^{1}$ College of Education and Sciences, Bohai University, Jinzhou, China \\ ${ }^{2}$ Key Laboratory of Emotion and Mental Health in Chongqing, Chongqing University of Arts and Sciences, Collaborative \\ Innovation Center for Brain Science, Chongqing, China \\ ${ }^{3}$ School of Psychology, The University of Newcastle, Newcastle, NSW, Australia \\ Email: chinaxiaofan@126.com, " gy8620@163.com, " ross.wilkinson@newcastle.edu.au
}

How to cite this paper: Fan, H., Ge, Y., \& Wilkinson, R. (2021). Humor Style and Creativity Tendency of Senior High School Students of Tujia Ethnic Group in China. Advances in Applied Sociology, 11, 141-157. https://doi.org/10.4236/aasoci.2021.114011

Received: January 27, 2021

Accepted: April 13, 2021

Published: April 16, 2021

Copyright $\odot 2021$ by author(s) and Scientific Research Publishing Inc. This work is licensed under the Creative Commons Attribution International License (CC BY 4.0).

http://creativecommons.org/licenses/by/4.0/

\begin{abstract}
To explore humor style and creativity tendency of senior high school students of Tujia, a Chinese ethnic group, and the relation between the two in Chinese ethnic culture, 127 Tujia high school students and 124 Han ones were selected to complete Humor Styles Questionnaire and Creativity Assessment Packet. The study questionnaires were administered in a classroom setting when participants were attending their classes. The results show that: 1) Tujia school students prefer affiliative and self-enhancing humor. Among them, urban male students and rural female students exhibit a greater tendency to engage in self-enhancing humor, while urban female students are relatively weak; first grade senior high school students from the city prefer maladaptive aggressive humor. Between Tujia and Han students, the former ones are more prone to engage in self-enhancing humor and less prone to engage in aggressive humor than their Han counterparts. 2) Creativity tendency of Tujia students is at a good level, especially inquisitiveness and adventure. In terms of overall performance and adventure, Tujia students fare better than the students of Han ethnicity, and then the first-grade students stand out among the Tujia students. 3) There is a significant positive correlation between humor style and creativity tendency in the Tujia groups. The affiliative humor style is associated with higher levels of creativity.
\end{abstract}

\section{Keywords}

Chinese Tujia Ethnic Group, Senior High School Students, Humor Style, Creativity Tendency

\footnotetext{
${ }^{\star}$ Co-first authors.

\#Co-corresponding authors.
} 


\section{Introduction}

Humor is a universal phenomenon present in all human groups and is associated with a myriad of factors. Although humor has been extensively researched and studied, it is the bisociation theory of Arthur Koestler (1964) that has directed researchers' attention to the relationship between humor and creativity. Humor exists in creative activities, but also the outstanding expression of creativity (Rudowicz \& Yue, 2001). Creativity is a core attribute that drives and supports social development and advancement. At this time when the world is grappling with COVID-19, creativity has been highlighting the importance of its own key competitiveness and vitality in response to the unprecedented crisis. Innovative education is the foundation of creativity cultivation. To promote the creativity and further the development of innovative education in the new era, it will be of great theoretical and practical significance to explore the links between humor and creativity of minority adolescents under the background of Chinese multi-ethnic culture.

\subsection{Humor}

The psychological components of humor include cognitive, emotional and behavioral elements among others. It is a specific set of stimuli perceived by individuals that can trigger funniness (Marin, 2000). Humor comprises the cognition of the funniness, contradiction and absurdity of perceptions and the positive evaluation of surroundings. It involves giving and taking happy emotions and staying amused. The most common and explicit expression of humor is laughter (Marin, 2001). A sense of humor refers to individual differences and habitual tendencies in behaviors, experiences, emotions, attitudes and abilities in humor-related activities (Ruch, 1998). The ways in which individuals engage in humor vary with different styles of humor having different impacts. Martin et al. (2003) proposed a widely adopted classification of humor styles. They classify humor into affiliative humor (the use of humor to enhance interpersonal relationships, reduce conflict and build cohesiveness), self-enhancing humor (having a good-natured attitude toward life and using humor to cope with stress without hurting others), aggressive humor (the use of criticism for self-enhancement at the expense of others) and self-defeating humor (inappropriate self-mockery and deprecation to gain approval from others). The former two styles are considered as adaptive and can potentially improve one's physical and psychological wellbeing, while the latter two are considered as maladaptive in that are potentially detrimental to one's wellbeing. Adaptive humor style is regarded as an important factor of the positive mental quality, which can alleviate individual psychological conflict (Chen \& Martin, 2007), improve mental resilience (Veselka et al., 2010; Fan \& Wang, 2020) and promote teamwork (Schfer, 2021). High agreeableness, openness and extraversion of personality traits were mainly related to the positive styles, as well as neuroticism correlated positively with sarcasm and criticism (Dionigi et al., 2021). 
Humor styles are, in part, a result of the different impacts of culture and traditions (Meng, 1993). Unlike the situation in Western countries, traditional Chinese humor seldom touches upon the subject of sex. Castell and Goldstein have found that Hong Kong students prefer witty and conservative jokes, while American students favor sex-related and aggressive jokes (Castell \& Goldstein, 1977); a comparative study of American and Singapore students' attitude toward humor by Nevo and others arrived at a similar conclusion (Nevo et al., 2001). Yue points out in his studies on Chinese humor that Chinese people are cautious, conservative, particular and often critical about the appreciation of humor. He refers to this as "serious humor," as opposed to humor for the sole purpose of entertainment in Western countries (Yue, 2008; Yue, 2010; Yue, 2014).

\subsection{Creativity}

Creativity is an individual's ability to produce novel and useful products or ideas in a specific environment and is an attribute inferred from the individual's demonstration of creative activities (Sternberg, 2000; Sternberg, 2005). A creativity tendency is associated with descriptors such as curiosity, imagination, challenge and adventure (Cai \& Zhu, 2007; Williams, 1980).

Creativity and culture are closely correlated with each other. Human creativity spurs cultural evolution and cultural diversity (Zhang \& Xiao, 2015); culture, in turn, acts as catalyst for human creativity by shaping certain individuals' or groups' values and ways of thinking (Kharkhurin \& Samadpour, 2008). Chinese nationals and Westerners, born and raised in different cultures, have different perceptions on creativity. To the Westerners, creativity represents inventiveness, originality and creative ability, which call for appreciation of beauty, artistic taste, ingenuity, sense of humor and critical thinking (Mayer, 1999). To the Chinese, however, creativity is driven largely by virtues, specifically the ethics and social responsibilities derived from collectivism (Shi \& Zhan, 2002; Yue \& Leung, 2003). In their eyes, creativity is synonymous with dedication and influence (Yue, 2001).

\subsection{Links between Humor and Creativity}

Studies on the relationship between humor and creativity have led to different conclusions. Integration and incongruity might coexist within the relation between humor and creativity (Murdock \& Ganim, 1993). It is universally acknowledged that humor and creativity are closely correlated. Highly creative people are thought to display humor even in face of problems and, likewise, and those with a strong sense of humor tend to be more creative (Glover, 1980). From the perspective of cognitive similarity, there is a common brain cognitive component between humor and insight (Nie \& Luo, 2012; Li, 2014). Humor and creativity both represent alternative ways of looking at situations (Wu \& Chen, 2019). Generally, positive humor, such as affiliative humor and self-enhancing humor, are considered more likely to inspire creativity versus negative humor 
styles (Ren, Chan, \& Chen, 2011). For example, Ziv (1976) find that participants in an experiment generally score higher in linguistic creativity after watching comedy shows. This might be because of the positive emotions associated with humor, which help to reduce processing time, save capacity and prompt lateral thinking, eventually inspiring creativity (Davis, 2009). A series of intervention studies conducted by Chen (2004) and Chen \& Hsu (2006) show that the creativity of the experimental group could be inspired and enhanced after taking humor courses. Moreover, another study further indicates that general humor endorsers have greater creativity (Chang et al., 2015). Other studies, however, have reached varied conclusions. Some scholars think that, in China, few people believe that humor was an essential trait. Anyhow, the Chinese are inferior to the Westerners in terms of sense of humor (Hao et al., 2007). Studies led by Yue \& Leung (2003) and Yue \& Xu (2011) suggest that university students in Chinese mainland, Taiwan and Hong Kong generally do not see humor as a vital element for creativity.

\subsection{Tujia Ethnic Group in China}

China is home to many different ethnic groups and a diverse range of minority cultures. The Tujia ethnic group, with a total population of about 8.35 million, is found mostly in Hunan, Hubei, Guizhou and the Wuling Mountains bordering Chongqing in China. Of the 55 ethnic minorities in China, the Tujia ethnic group is the seventh largest (NEAC, 2020). One of the largest ethnic groups in southwestern China, Chongqing is now home to about 1.4 million of the Tujia people, more than any other ethnic minority in Chongqing (Peng, 2011). Tujia people are accustomed to worshiping ancestors, are polytheistic, and are generally considered as typically optimistic, persistent and generous (Peng, 2012). Currently, studies on the adolescent of Tujia ethnic minority have largely centered on interpersonal violence, ethnic identity, alienation, attachment and self-esteem, sexual knowledge and attitudes, psychological conditions of autism, addiction and other health-risk behaviors (Qin \& Lu, 2010; Gao et al., 2012; Ceng, 2008; Kang, 2008; Ge \& Zhao, 2012; Zhang \& Xu, 2013; Zhang et al., 2015; Zhang \& Xiao, 2015; Zheng, 2016; Yang, 2016). None, so far, has been conducted on the relationship between humor style and creativity tendency in this particular group.

To sum up, past research results on the relationship between humor, creativity and culture hold for various ethnic groups in China, particularly among the adolescents from the ethnic groups in western China, still needs verification. It is also the crucial part of in-depth implementation of innovation-driven development to explore the status quo and characteristics of ethnic minorities so as to motivate their innovative vitality and creative potential.

With a view to this, the current study was carried out on senior high school students of Tujia ethnicity, and designed to explore the relationship between humor style and creativity tendency among this group influenced by local culture. This will enhance research on the relationship between humor style and 
creativity tendency across different ethnic groups and provide empirical evidence for the psychological studies on adolescents in ethnic minorities.

\subsection{Hypotheses}

Hypothesis 1: There are differences in humor styles between the senior high school students from the Tujia and Han ethnic groups, and among the Tujia students.

Hypothesis 2: It exists differences in creativity tendency between the senior high school students from the Tujia and Han ethnic groups, and among the $\mathrm{Tu}$ jia students.

Hypothesis 3: There is a significant correlation between the humor style and creativity tendency of the Tujia senior high school students.

\section{Methods}

\subsection{Participants}

Volunteer students of Tujia and Han ethnic backgrounds from five high schools in Youyang, Xiushan and Qianjiang, four Tujia autonomous counties, of Chongqing in China were recruited as participants through posters and flyers approved by Chongqing University of Arts and Sciences Institutional Review Board. Both researchers and participants signed the Safety Agreement and the Informed Consent Form. A total of 300 questionnaires were distributed and 251 were collected on site, including 127 Tujia respondents and 124 Han respondents. See Table 1 for detailed research objects.

\subsection{Measures}

\subsubsection{Humor Style Questionnaire, HSQ}

The Humor Style Questionnaire (HSQ) produced by Martin et al. (2003) and translated and revised by Chen \& Martin (2007) was employed. This measure includes 25 items assessing four dimensions: affiliative humor (8 items), self-enhancing humor (5 items), aggressive humor (7 items) and self-defeating humor (5 items). A seven-point Likert scale specified respondents' level of agreement or disagreement and for some items, reverse scoring was applied as appropriate. Higher scores suggest a stronger tendency of certain humor style. In this study, Cronbach's alphas of the four-dimension questionnaires are $0.81,0.78,0.61$ and 0.72 respectively, and retest reliability coefficients are $0.57-0.67$.

Table 1. Research objects.

\begin{tabular}{cccc}
\hline Grade & Grade 1 & Grade 2 & Grade 3 \\
\hline Gender (Male/Female) & $46 / 43$ & $34 / 42$ & $40 / 46$ \\
Place of Residence (Rural/Urban) & $34 / 55$ & $38 / 38$ & $31 / 55$ \\
Ethnic Group (Tujia/Han) & $49 / 40$ & $40 / 36$ & $38 / 48$ \\
Total & 89 & 76 & 86 \\
\hline
\end{tabular}




\subsubsection{Creativity Assessment Packet, CAP}

Produced by American psychologist Williams (1993) and revised by Lin and Wang (1994), the Creativity Assessment Packet (CAP) contains 50 items, which assess adventure (11 items to assess whether one has the courage to face failure or criticism, dares to guess, and can complete tasks in a messy situation and defend one's own point of view), curiosity (14 items to assess whether one has the spirit of exploration and various ideas, is willing to approach ambiguous situations, thinks deeply about things, and can grasp special phenomenon), imagination (13 items to assess whether one can visualize and create mental images, imagine things that have not yet happened, intuitively speculate and transcend the limitations of perception and reality) and challenging (12 items to assess whether one can explore possibilities, understand the gap between possibility and reality and bring order out of chaos, and is willing to explore complex questions and ideas). The three-point response format for the CAP was adopted (totally disagree $=1$, partially agree $=2$, totally agree $=3$ ), and items were reverse scores as appropriate. Higher scores indicate a stronger tendency for creativity. A total score over 133 indicates outstanding creativity, $111-133$ is good and under 111 normal (Lin \& Wang, 1994). CAP is widely used in countries all over the world, suitable for children and adolescents with good reliability and validity. In this study, Cronbach's alphas of the overall and four-dimension questionnaires are $0.82,0.73,0.76,0.71$ and 0.69 respectively.

SPSS 20.0 was used for data processing to conduct $t$ test, test for homogeneity of variance, correlation analysis and regression analysis.

\section{Results}

\subsection{General Traits of Humor Styles of High School Students of Tujia Ethnic Group}

Table 2 illustrates that high school students of Tujia ethnic group get the highest scores in affiliative humor and the lowest scores in self-defeating humor. The score ranking, from high to low, is affiliative humor, self-enhancing humor, aggressive humor, self-defeating humor.

To explore the influence on humor styles of high school students of Tujia ethnic group by gender, grade and place of residence, multi-factor analysis of variance (multi-factor ANOVA) was conducted to analyze the main effect and interaction of gender, grade and place of residence. The result indicates that self-enhancing humor has a significant interaction with gender and place of

Table 2. General traits of humor styles of Tujia students $(\mathrm{N}=127)$.

\begin{tabular}{cccccc}
\hline & N & Minimum & Maximum & Average & Standard deviation \\
\hline Affiliative humor & 127 & 12.00 & 55.00 & 36.15 & 8.60 \\
Self-enhancing humor & 127 & 7.00 & 37.00 & 15.63 & 6.29 \\
Aggressive humor & 127 & 6.00 & 35.00 & 22.50 & 6.49 \\
Self-defeating humor & 127 & 5.00 & 28.00 & 13.15 & 5.20
\end{tabular}


residence. The interactive relationship is significant, with $F(1,115)=1.024, p<$ 0.05 . Aggressive humor has conspicuous interactive relationship with grade and place of residence, with $F(2,115)=1.048, p<0.05$.

A simple effect test of interaction relationship and Post Hoc test of three or more factors show that: there is a significant urban-rural difference in senior high school female students' self-enhancing humor $(p<0.05)$, and rural girls are of higher self-enhancing type than urban girls; there is an obvious gender difference in urban senior high school students' self-enhancing humor $(p<0.01)$, and boys are of higher self-enhancing type than girls. There is a clear urban-rural difference in aggressive humor of grade one high school students of Tujia ethnic group, and urban students are of higher aggressive type than rural students $(p<0.01)$ (Table 3$)$.

Table 4 suggests that, there is a significant difference between high school students of Tujia ethnic group and high school students of Han ethnic group in the scores of affiliative humor $(p<0.01)$, and high school students of Tujia ethnic group get higher scores; there is also an obvious difference between them in the scores of aggressive humor $(p<0.05)$, and high school students of Han ethnic group obtain higher scores. In the other two humor styles, there is no remarkable difference between high school students of Tujia ethnic group and high school students of Han ethnic group.

\subsection{Characteristics of Creativity Tendency of High School Students of Tujia Ethnic Group}

Table 5 shows that the average score of creativity tendency of high school students of Tujia ethnic group is 128.77 , which is at a good level. They get highest scores in curiosity and lowest scores in imagination. The score ranking, from high to low, is curiosity, adventure, challenging, imagination.

Table 3. Differences of variables of humor styles of Tujia students.

\begin{tabular}{|c|c|c|c|c|c|}
\hline \multirow{3}{*}{$\begin{array}{l}\text { Place of } \\
\text { residence }\end{array}$} & \multirow{2}{*}{\multicolumn{2}{|c|}{$\begin{array}{c}\text { Self-enhancing humor }(M \pm S D) \\
\text { Gender }\end{array}$}} & \multicolumn{3}{|c|}{ Aggressive humor $(M \pm S D)$} \\
\hline & & & & Grade & \\
\hline & Male & Female & Senior 1 & Senior 2 & Senior 3 \\
\hline Urban & $17.18 \pm 7.07$ & $13.90 \pm 4.77$ & $22.86 \pm 6.94$ & $21.76 \pm 6.83$ & $22.86 \pm 6.94$ \\
\hline Rural & $17.14 \pm 7.12$ & $17.90 \pm 7.19$ & $15.02 \pm 6.74$ & $19.67 \pm 5.78$ & $19.37 \pm 6.28$ \\
\hline
\end{tabular}

Table 4. Comparison of difference of humor styles between Tujia and Han students.

\begin{tabular}{cccc}
\hline & Tujia Students (127) & Han Students (124) & \multirow{2}{*}{$t$} \\
\cline { 2 - 3 } & $M \pm S D$ & $M \pm S D$ & \multirow{2}{*}{$\mathbf{3 . 0 9 1 ^ { * * }}$} \\
\hline Affiliative humor & $37.40 \pm 7.07$ & $30.26 \pm 4.78$ & 0.334 \\
Self-enhancing humor & $22.50 \pm 6.49$ & $22.22 \pm 7.08$ & $-\mathbf{2 . 3 0 6 ^ { * }}$ \\
Aggressive humor & $15.63 \pm 6.29$ & $17.64 \pm 7.45$ & -0.196 \\
Self-defeating humor & $13.15 \pm 1.20$ & $13.28 \pm 5.53$ & -0.196 \\
\hline
\end{tabular}

Note: ${ }^{\star}: p<0.05 ;{ }^{* *}: p<0.01{ }^{* * *}: p<0.001$. 
Table 5. General traits of creativity tendency of Tujia students $(\mathrm{N}=127)$.

\begin{tabular}{cccccc}
\hline & $\mathrm{N}$ & Minimum & Maximum & Average & Standard deviation \\
\hline Adventure & 127 & 22.00 & 40.00 & 30.56 & 3.51 \\
Curiosity & 127 & 21.00 & 49.00 & 36.83 & 5.26 \\
Imagination & 127 & 17.00 & 37.00 & 28.84 & 5.07 \\
Challenging & 127 & 18.00 & 43.00 & 29.80 & 3.53 \\
Total scores & 127 & 97.00 & 163.00 & 128.77 & 13.72 \\
\hline
\end{tabular}

Multi-factor ANOVA of gender, grade and place of residence indicates that grade has a main effect on the total scores of creativity tendency in adventure, with $F(1,115)=3.92, p<0.05 ; F(1,115)=4.52, p<0.05$. The main effect of senior one is greater than senior three, and senior three greater than senior two. There was no other difference (Table 6).

Table 7 suggests that, there is a significant difference between high school students of Tujia ethnic group and high school students of Han ethnic group in the scores of risk taking $(p<0.05)$, and high school students of Tujia ethnic group get higher scores than those of Han ethnic group. In the other two creativity tendencies, there is no remarkable difference between high school students of Tujia ethnic group and high school students of Han ethnic group.

\subsection{Humor Style and Creativity Tendency of High School Students of Tujia Ethnic Group}

Table 8 shows that there is an obvious positive correlation between humor style and creativity tendency of Tujia high school students. More specifically, a positive correlation exists between affiliative humor and total scores of creativity, curiosity, imagination and challenging; a positive association is also linked across self-defeating humor, total scores of creativity and imagination.

To further explore the influence of humor styles on creativity tendency, on the basis of above relevant findings, regression analysis was made, with humor styles as independent variables and creativity tendency as dependent variable. The result reveals that affiliative humor in the regression equation can positively predict creativity tendency, variance explained reaching 50\% (Table 9).

\section{Discussion}

\subsection{Traits of Humor Styles of Senior High School Students of Tujia Ethnic Group}

The study shows that the humor styles of senior high school students of Chinese Tujia ethnic group are generally affiliative and self-enhancing. Some researchers conclude that Tujia people are inclusive, innovative, cohesive and cooperative (Liang, 2005; Chen, 2000); and they feature the personality characteristics of positive, intimate and collaborative agreeableness (Peng et al., 2014). All this is consistent with the improvement in coordination and cohesion of affiliative and 
Table 6. Comparison of difference of creativity tendency of Tujia students.

\begin{tabular}{|c|c|c|c|c|c|}
\hline & Senior 1 & Senior 2 & Senior 3 & \multirow{2}{*}{$F$} & \multirow{2}{*}{ Multiple comparisons } \\
\hline & $M \pm S D$ & $M \pm S D$ & $M \pm S D$ & & \\
\hline Total scores & $26.55 \pm 3.11$ & $24.98 \pm 2.42$ & $25.55 \pm 2.34$ & $3.92^{*}$ & $\begin{array}{l}\text { senior } 1>\text { senior } 3 \\
\text { senior } 3>\text { senior } 2\end{array}$ \\
\hline Adventure & $6.33 \pm 0.82$ & $3.11 \pm 0.70$ & $5.91 \pm 0.59$ & $4.52^{*}$ & $\begin{array}{l}\text { senior } 1>\text { senior } 3 \text {, } \\
\text { senior } 3>\text { senior } 2\end{array}$ \\
\hline
\end{tabular}

Note: ${ }^{*}: p<0.05 ;{ }^{* *}: p<0.01 ;{ }^{* *}: p<0.001$.

Table 7. Comparison of difference of creativity tendency between Tujia and Han students.

\begin{tabular}{cccc}
\hline & Tujia Students (127) & Han Students (124) & \multirow{2}{*}{$t$} \\
\cline { 2 - 3 } & $M \pm S D$ & $M \pm S D$ & \\
\hline Total scores & $129.06 \pm 16.74$ & $128.77 \pm 13.73$ & $\mathbf{0 . 1 5 1 ^ { * }}$ \\
Adventure & $30.75 \pm 4.64$ & $30.56 \pm 3.51$ & $\mathbf{0 . 3 6 7 ^ { * }}$ \\
Curiosity & $37.38 \pm 5.84$ & $36.83 \pm 5.26$ & 0.776 \\
Imagination & $28.84 \pm 5.07$ & $28.51 \pm 6.03$ & 0.464 \\
Challenging & $29.80 \pm 3.53$ & $29.63 \pm 4.10$ & 0.361 \\
\hline
\end{tabular}

Note: ${ }^{*}: p<0.05 ;{ }^{* *}: p<0.01 ;{ }^{* *}: p<0.001$.

Table 8. Correlation between humor style and creativity tendency of Tujia students.

\begin{tabular}{|c|c|c|c|c|c|}
\hline & Total scores & Adventure & Curiosity & Imagination & Challenging \\
\hline Affiliative humor & $0.223^{*}$ & 0.046 & $0.240^{* *}$ & $0.177^{\star}$ & $0.188^{*}$ \\
\hline Self-enhancing humor & 0.091 & 0.032 & 0.155 & -0.037 & 0.152 \\
\hline Aggressive humor & 0.089 & 0.030 & 0.049 & 0.034 & 0.095 \\
\hline Self-defeating humor & $0.182^{*}$ & 0.092 & 0.170 & $0.192^{*}$ & 0.041 \\
\hline
\end{tabular}

Note: "total scores" refers to the total scores of creativity tendency questionnaires. ${ }^{*}: p<0.05$; ${ }^{* *}: p<0.01$; ${ }^{* * *}: p<0.001$.

Table 9. Regression analysis on the humor style and creativity tendency of Tujia students.

\begin{tabular}{lccccc}
\hline Dependent Variable & Independent Variable & $R^{2}$ & $\beta$ & $F$ & $t$ \\
\hline Creativity tendency & Affiliative humor & 0.50 & 0.22 & $\mathbf{6 . 5 7}^{*}$ & $2.56^{*}$ \\
\hline
\end{tabular}

Note: ${ }^{*}: p<0.05 ;{ }^{* *}: p<0.01 ;{ }^{* *}: p<0.001$.

self-enhancing humor styles. Therefore, senior high school students of Tujia ethnic group are generally good at maintaining a good relationship with others and identify less with the self-defeating humor.

For Tujia students, their self-enhancing humor has an interactive effect on gender and place of residence. Tujia urban males score higher than females, which is in accordance with the study result of Canadian samples of Martin et al. (2003). Additionally, from the research of Wu et al. (2016), there are also some findings regarding Chinese young male adolescents' dominant role in self-enhancing 
humor. The traditional gender roles require that men be independent, progressive, earnest, responsible, brave and ambitious. Broadly, Tujia culture highlights optimism, progressiveness, boldness and responsibility (Feng, 2005). When it comes to gender roles, the Tujia ethnic group leans towards traditional values, advocating respect for seniority and clear labor division between men and women (Zhou \& Xiao, 2013). According to the post-interviews with students and parents, it's known that the sexual division of labor plays a critical role in family education of Tujia children. Affected by traditional gender roles and the overall ethnic personalities, urban Tujia boys may be inclined to use the self-enhancing humor to cope with difficulties and pressures. On the other hand, rural Tujia girls score higher than those in cities and towns regarding self-enhancing humor. This may be because Tujia girls predominantly live in mountainous areas, mostly in cavern-connected high hills, large rivers and bushes, and need "brave" and "simple" spirits as well as the self-enhancing humor to lead their lives (Huang, 2011; Cui, 2014). In comparison, there is the significant urban-rural gap in China, and cities have better living environment, learning opportunities and conditions. This decides that urban Tujia girls can get rid of such original environment to enjoy a better family environment and emotional care as well as a richer life. As a result, their consciousness of independence is slightly less strong.

In addition, the grades and places of residence were found to have an interaction effect on aggressive humor. First-year students of senior high schools in cities and towns prefer the aggressive humor, which is in accord with the results of study on Canadian samples by researchers including Martin and Wang's research of senior high school students of the Chinese Wa ethnic group (Marin, 2001; Wang, 2013). In China, first-year students need to adapt to new environment and establish new relationships while entering senior high schools. Driven by anxiety and uncertainty, they usually adopt the aggressive humor to display themselves, impress others as well as gain recognition and acceptance. The maladaptive humor style mostly occurs in certain scenarios and doesn't run counter to the overall adaptive humor of Tujia senior high school students. They will outgrow the aggressive humor style.

Compared with students of Han ethnic group, Tujia students report more self-enhancing humor than the aggressive humor. This result is consistent with the general characteristics of Tujia senior high school students' humor styles.

\subsection{Traits of Creativity Tendency of Senior High School Students of Tujia Ethnic Group}

The present studies results indicate that the creativity tendency of senior high school students of Tujia ethnic group is at a good level, characterized mainly by inquisitiveness and adventure. In terms of the overall level and adventure, students of Tujia ethnic group outperform students of Han ethnic group. Within students of Tujia ethnic group, grade one high school students are more prominent. 
Living in high mountains and deep rivers, Tujia ethnic group leads a challenging life. But their ancestors' optimism and open-mindedness make for an active, open-minded and progressive nationality (Zhou \& Xiao, 2013; Xiao \& Zhang, 2015). When facing the cultures of Han people, Tujia ethnic group takes an open attitude to absorb these cultures and enrich themselves while inheriting and promoting their own cultures and traditions. Tujia people have a way of using exaggeration, imagination and ironic remarks to cope with difficulties in daily lives and social struggles, as well as to find amusement. Their imagination is exaggerative and bold, revealing their critical thinking (Peng et al., 2014).

Teenagers' creativity tendency changes over time. Their creativity is on an upward trajectory from primary schools to junior high schools and starts to level off in senior high schools. Junior high schools are a critical stage when teenagers move from adolescence to adulthood. During this period, with the pursuit of novelty, their self-awareness increases. After entering senior high schools, a slew of issues such as further study would force Chinese students to be realistic. They have to manage to conform to social regulations and common standards, which would slow down the development of creativity (Cai \& Zhu, 2007). First-year senior high school students of Tujia ethnic group still have the thinking modes and psychological characteristics of junior high schools. New environment evokes their anxiety but also curiosity and risk-taking spirit. Due to a large population in China, the competition in education is always fierce. Entering higher levels of education, especially the national college entrance examination, will constantly test the endurance of adolescents. Therefore, as study pressure increases, Tujia students would start to restrain themselves to move towards the goal of college entrance examination. In interviews, students in senior grades point out that college entrance examination provides a more important practical significance for them than for students of Han ethnic group. Compared with Han students, minority students cannot enjoy the same quality teaching, environment and opportunities. Parents and teachers repeatedly exhort them to cherish learning opportunities and avoid being too playful and risk-taking.

\subsection{Humor Style and Creativity Tendency of Senior High School Students of Tujia Ethnic Group}

There is a significant positive correlation between humor style and creativity tendency of senior high school students of Tujia ethnic group. The affiliative humor style can positively predict creativity tendency. This indicates that the affiliative humor style plays an important role in enhancing creativity of Tujia senior high school students, showing distinctive national characteristics. The affiliative humor style is a well-accepted way of humor expression, which can strengthen group cohesion and contribute to harmonious human relationships, and moreover, greater activation can be observed in neural correlates involved in the affiliative humor (Chan et al., 2018). Adaptive humor can help to stimulate euphoric feelings. Positive emotion is beneficial to widening individual cognitive range and speeding up information processing, thus facilitating the development 
of creativity (Lu et al., 2002; Hu \& Wang, 2010; Davis, 2009). Individuals good at using adaptive humor can break the inherent logical relations in a happy, relaxing way and achieve innovative and ground-breaking thinking to establish novel significance and connection. When things are more unexpected, curiosity will be satisfied to a greater extent and stronger risk-taking spirit and imagination will be encouraged (Xu, 2002; Zhang \& Shang, 2019).

\section{Conclusion}

High school students of Tujia ethnic group are mainly of adaptive humor style including affiliative type and self-enhancing type. In terms of internal difference, urban boys and rural girls are more of self-enhancing type than urban girls. First grade urban high school students appear to prefer a hostile (i.e., non-adaptive) humor style. In terms of external difference, compared with students of Han ethnic group, students of Tujia ethnic group are of higher self-enhancing type and lower hostile type.

The creativity tendency of high school students of Tujia ethnic group is at a relatively high level, mainly characterized by inquisitiveness and adventure. Regarding the overall level and adventure, students of Tujia ethnic group are higher than students of Han ethnic group. Within students of Tujia ethnic group, grade one high school students are most prominent.

There is a significant positive correlation between humor style and creativity tendency of high school students of Tujia ethnic group. The affiliative type humor style can positively predict creativity tendency.

\section{Enlightenment and Expectation}

Innovation ability is one of the core characteristics of talents in the new century. Furthermore, high school stage is a key period for pushing the individuals to be more innovative and quick-thinking. Thus, the characteristics of the relationship between the humor style and creativity of Tujia high school students and the unique cultural environment have been providing some important enlightenment for the cultivation of innovation ability of minority adolescents in current creative educational practice.

On the basis of positive emotions, humor can directly express creativity and develop the ability of personal innovation. Therefore, creating a humorous environment and promoting the development of adaptive humor for Tujia high school students, as well as taking maximum advantage of humor to reduce anxiety, relax mood and release pressure, will be furthering their mental health level.

The fostering of creativity has its cultural roots. The more diverse, open and free a culture is, the more abundant, dynamic and competitive its creativity will be, and the greater its role in culturing creativity will be then. So, rooted in the inclusive and free national culture of Tujia people, the overall creativity level of Tujia adolescents will be advanced by absorbing a wide range of cultural nu- 
trients.

\section{Conflicts of Interest}

The author(s) declared no potential conflicts of interest with respect to the research, authorship, and/or publication of this article.

\section{Ethical Approval}

This article does not contain any studies with animals performed by any of the authors. All procedures performed in studies involving human participants were in accordance with the ethical standards of the institutional and national research committee and with the 1964 Helsinki declaration and its later amendments.

\section{Informed Consent}

All participated in the study voluntarily. The researchers obtained approval from the participants.

\section{References}

Cai, X. Y., \& Zhu, Y. J. (2007). The Correlation of Adolescent's Creative Inclination, Intelligence and Academic Achievement. Psychological Development and Education, 23, 36-41.

Castell, P. J., \& Goldstein, J. H. (1977). Social Occasions of Joking: A Cross-culture Study. In A. J. Chapman, \& H. C. Foot (Eds.), It's a Funny Thing Humor (pp. 193-197). Oxford: Pergamon Press. https://doi.org/10.1016/B978-0-08-021376-7.50040-1

Ceng, X. Z. (2008). Investigation and Study on the Alienation of Southeast Minority Adolescent Students. Chongqing: Southwest University.

Chan, Y. C., Hsu, W.-C., Liao, Y. J., Chen, H. C., Tu, C. H., \& Wu, C. L. (2018). Appreciation of Different Styles of Humor: An fMRI Study. Scientific Reports, 8, Article No. 15649. https://doi.org/10.1038/s41598-018-33715-1

Chang, J. H., Chen, H. C., Hsu, C. C., Chan, Y. C., \& Chang, Y. L. (2015). Flexible Humor Styles and the Creative Mind: Using a Typological Approach to Investigate the Relationship between Humor Styles and Creativity. Psychology of Aesthetics, Creativity, and the Arts, 9, 306-312. https://doi.org/10.1037/a0039527

Chen, G. A. (2000). Tujia National Spirit Probe. Guizhou Ethnic Studies, No. 2, 91-97.

Chen, G. H., \& Martin, R. A. (2007). Humor Styles and Mental Health among Chinese University Students. Psychological Science, 30, 219-223.

Chen, H. C. (2004). Evaluating the Influence of the Training Curriculum ICEMB (Integrating Cognition, Emotion, Motivation, and Behavior) to College Students' Sense of Humor and Creativity. Bulletin of Educational Psychology, 35, 393-411.

Chen, H. C., \& Hsu, C. C. (2006). Evaluating the Impact of the Humor Training Curriculum on Teachers' Sense of Humor and Creativity. Journal of National Taiwan Normal University: Education Edition, 51, 71-93.

Cui, Y. L. (2014). The Variation of Ethnic Minority Women's Self in Cultures Exchange-Taking Enshi Tujia Shuanglong Village as an Example. Journal of Huazhong University of Science and Technology (Social Science Edition), 28, 110-117. 
Davis, M. A. (2009). Understanding the Relationship between Mood and Creativity: A Meta-Analysis. Organizational Behavior and Human Decision Processes, 108, 25-38. https://doi.org/10.1016/j.obhdp.2008.04.001

Dionigi, A., Duradoni, M., \& Vagnoli, L. (2021). Humor and Personality: Psychometric Properties of the Italian Version of the Comic Styles Markers and Its Relationships with the Big Five Personality Traits. Current Psychology, Advance Online Publication. https://doi.org/10.1007/s12144-020-01303-0

Fan, R., \& Wang, B. (2020). Relationship between Self-Enhancing Humor Style and Athletes' Mental Toughness: The Mediating Effect of Sense Making of Adversity. Journal of Tianjin University of Sport, 35, 356-360.

Feng, Z. M. (2005). A Perspective of the National Psychology of the Tujia Living Culture of Youshui in the Eastern Chongqing. Journal of Chongqing Institute of Socialism, No. 4, 63-65.

Gao, Y. D., Zhang, J. F., Gong, X. et al. (2012). The Relationship between National Identity and Self-Esteem among Chinese Tujia University Students. Health Care Research and Practice, 9, 27-30.

Ge, Y., \& Zhao, Y. (2012). Study on the Compilation and Characteristics of Family Junior Middle School Students' Sexual Knowledge and Sexual Attitude Questionnaire. Chinese Journal of Health Statistics, 29, 826-829.

Glover, J. A. (1980). Becoming a More Creative Person. Englewood Cliffs, NJ: Prentice Hall.

Hao, X., Yue, X. D., Qi, S. S., \& Qi, S. Y. (2007). Investigation and Reflection on Sense of Humor of Chinese University Students. Journal of Inner Mongolia Normal University (Natural Science Edition), 36, 33-36.

Hu, W. P., \& Wang, X. Q. (2010). The Effect of Mood on Problem Finding in Scientific Creativity. Psychological Science, 42, 608-611.

Huang, X. R. (2011). Research on the Life and Social Gender of Tujia Women in Historical Period. Chongqing: Southwest China Normal University Press.

Kang, L. (2008). Characteristics and the Relationship of the Attachment and Self-esteem of Middle School Students of Tujia Ethnic Group. Nanchang: Jiangxi Normal University.

Kharkhurin A. V., \& Samadpour Motalleebi, S. N. (2008). The Impact of Culture on the Creative Potential of American, Russian and Iranian College Students. Creativity Research Journal, 20, 404-411. https://doi.org/10.1080/10400410802391835

Koestler, A. (1964). The Act of Creation. Oxford: Macmillan.

Li, Y. (2014). Neurocognitive Mechanisms and Influencing Factors of Semantic Insight Problem Solving. Wuhan: Central China Normal University.

Liang, Y. B. (2005). Lovely Xiangxi: National Traits of Tujia \& Miao Ethnic Groups. Changsha: Hunan People's Publishing House.

Lin, H. T., \& Wang, M. J. (1994). Creativity Assessment Packet (CAP): Manual. Taipei: Psychological Publishing Co., Ltd.

Lu, J. M., Liu, W., He, W. et al. (2002). Effects of Mood States on Creativity. Acta Psychologica Sinica, 34, 381-386.

Marin, R. A. (2000). Humor. In A. E. Kazdin (Ed.), Encyclopdia of Psychology (Vol. 4). Washington DC: American Psychology Association. New York: Oxford.

Marin, R. A. (2001). Humor, Laughter, Physical Health: Methodological Issues and Research Findings. Psychological Bulletin, 127, 504-519. 
https://doi.apa.org/doi/10.1037/0033-2909.127.4.504

Martin, R., Puhlik-Doris, P., Larsen, G., Gray, J., \& Weir, K. (2003). Individual Differences in Uses of Humor and Their Relation to Psychological Well-Being: Development of the Humor Styles Questionnaire. Journal of Research in Personality, 37, 48-75.

https://doi.org/10.1016/S0092-6566(02)00534-2

Mayer, R. E. (1999). Fifty Years of Creativity Research. In R. J. Sternberg (Ed.), Handbook of Creativity (pp. 449-460). Cambridge: Cambridge University Press. https://doi.org/10.1017/CBO9780511807916.024

Meng, Z. Q. (1993). Humor and Culture. Zhengzhou: Henan People's Publishing House (HPPH).

Murdock, M. C., \& Ganim, R. M. (1993). Creativity and Humor: Integration and Incongruity. The Journal of Creative Behavior, 27, 57-70. https://doi.org/10.1002/j.2162-6057.1993.tb01387.x

NEAC (National Ethnic Affairs Commission of the People's Republic of China) (2020). Tujia Ethnic Group. http://www.seac.gov.cn/seac/ztzl/tjz/gk.shtml

Nevo, O., Nevo, B., \& Leong Siew Yin, J. (2001). Singaporean Humor: A Cross-Cultural, Cross-Gender Comparison. Journal of General Psychology, 128, 143-156. https://doi.org/10.1080/00221300109598904

Nie, Q. Y., \& Luo, J. (2012). “Aha!” and “Haha!”: The Common and Distinct Cognitive Brain Processes Underlying Insight and Humor. Advances in Psychological Science, 20, 219-227. https://doi.org/10.3724/SP.J.1042.2012.00219

Peng, F. R. (2011). Study on Ethnic Minorities living in Chongqing: Tujia Volume. Chongqing: Chongqing Publishing House.

Peng, F. R., Liu, L., Hui, Z., \& Liu, P. (2014). Tujia Personality Characteristics of the "Big Five Personality" Traits. Journal of Chongqing Three Gorges University, No. 5, 39-44.

Peng, W. L. (2012). Tujia Ethnic Group of China. Yinchuan: Ningxia People’s Publishing House.

Qin, S., \& Lu, Q. (2010). Correlation Analysis of Interpersonal Violence Tendency and Psychological Status on Tujia College Student. Journal of Hubei University for Nationalities (Medical Edition), 27, 17-19.

Ren, Y. Y., Chan, Y. C., \& Chen, H. C. (2011). The Relationship between Humor Styles and Creativity of Junior High School Students. New Horizons in Education, 39, 145-153.

Ruch, W. (1998). The Sense of Humor: Explorations of a Personality Characteristic. New York, NY: Mouton de Gruyter.

Rudowicz, E., \& Yue, X. D. (2001). Concepts of Creativity: Similarities and Difference among Hong Kong, Mainland and Taiwanese Chinese. The Journal of Creative Behavior, 34, 175-192. https://doi.org/10.1002/j.2162-6057.2000.tb01210.x

Schfer, J. (2021). Humor-Klebstoff für Teams. In S. Jessica (Ed.), Altersgemischte Teams in der Pflege (pp. 191-196). Berlin, Heidelberg: Springer.

https://doi.org/10.1007/978-3-662-62062-5 18

Shi, J., \& Zhan, Z. (2002). Psychological Research on and Education of Gifted and Talented Children in China. In K. A. Heller (Ed.), International Handbook for Research on Gifted and Talented (p. 22). Cambridge: Cambridge University Press.

Sternberg, R. J. (2000). Handbook of Intelligence. Cambridge: Cambridge University Press. https://doi.org/10.1017/CBO9780511807947

Sternberg, R. J. (2005). Handbook of Creativity. Beijing: Beijing Institute of Technology 
Press.

Veselka, L., Schermer, J. A., Martin, R. A., \& Vernon, P. A. (2010). Laughter and Resiliency: A Behavioral Genetic Study of Humor Styles and Mental Toughness. Twin Research \& Human Genetics, 13, 442-449. https://doi.org/10.1375/twin.13.5.442

Wang, X. (2013). Cross-Cultural Study on the Relationship between Humorous Style and Emotional Creativity of Wa and Han High School Students. Kunming: Yunnan Normal University.

Williams, F. E. (1980). Creativity Assessment Packet (CAP): Manual. Buffalo: D.O.K. Publishers, Inc.

Williams, F. E. (1993). Creativity Assessment Packet (CAP): Examiner's Manual. Austin, TX: Pro-Ed.

Wu, C. L., \& Chen, H. C. (2019). The Influence of Creativity on Incongruity-Resolution and Nonsense Humor Comprehension. Creativity Research Journal, 31, 110-118. https://doi.org/10.1080/10400419.2019.1577675

Wu, C. L., Lin, H. Y., \& Chen, H. C. (2016). Gender Differences in Humour Styles of Young Adolescents: Empathy as a Mediator. Personality and Individual Differences, 99, 139-143. https://doi.org/10.1016/j.paid.2016.05.018

Xiao, H. E., \& Zhang, W. Z. (2015). The Elaboration for Tujia Philosophy. Guangzhou: World Publishing Corporation.

Xu, C. Y. (2002). Creativity and Sense of Humor. Beijing: China Building Materials Industry Press.

Yang, Q. (2016). The Analysis of the Relationship between the Risking Behavior of Health of the High School Students in Rural of Xiang Xi Autonomous Prefecture and their Personality Traits. Jishou: Jishou University.

Yue, X. D. (2001). A Comparative Study among University Students in Beijing, Guangzhou, Hong Kong and Taipei. Acta Psychologica Sinica, 33, 148-154.

Yue, X. D. (2008). The Chinese Attitudes to Humor: Views from Undergraduates in Hong Kong and China. Educational Research Journal, 23, 299-325. (In Chinese )

Yue, X. D. (2010). Exploration of Chinese Humor: Historical Review, Empirical Findings, and Critical Reflections. Humor, 23, 403-420. https://doi.org/10.1515/humr.2010.018

Yue, X. D. (2014). The Attitudes towards Humor of Confucian, Buddhist, and Taoist Culture. Psychological Exploration, 34, 15-18.

Yue, X. D., \& Leung, K. (2003). Motives and Attitudes for Creativity: Views from Undergraduates in Hong Kong and Guangzhou. New Horizons in Education, 47, 1-5. (in Chinese)

Yue, X. D., \& Xu, N. N. (2011). The Relationship Between Humor Styles and Creativity, Survey of 163 Hong Kong University Students. New Horizons in Education, 59, 137-144.

Zhang, F. L., Zhang, T. C., Lu, S. H. et al. (2015). Analysis on Influencing Factors of Health Risk Behaviors of Wuling Mountainous Tujia and Miao adolescents. Chinese Journal of School Health, 36, 352-356.

Zhang, F. L., Zhang, T. C., Xiong, J. M. et al. (2015). Adolescent Addictive Behavior and its Influencing Factors of Wuling Mountainous Tujia and Miao adolescents. Chinese Journal of Public Health, 31, 1381-1385.

Zhang, L., \& Xu, G. C. (2013). Psychological Behavior Characteristics and Diagnosis of Miao and Tujia Autistic Children. Maternal and Child Health Care of China, 28, 462-463. 
Zhang, W. S., \& Xiao, Q. S. (2015). Studies on Culture and Creativity Cultivation. Educational Research, No. 5, 13-19.

Zhang, Y. J., \& Shang, G. Q. (2019). Research on Leader's Humor Style Influencing Employee's Creativity. Leadership Science, No. 19, 94-99.

Zheng, G. (2016). The Research on Middle Rural School Students' Ignore the Status and Health Risk Behavior of the Tujia and Miao Nationality in Western Hunan Province. Jishou: Jishou University.

Zhou, X. M., \& Xiao, Y. (2013). Theory on Primary Characteristics of Tujia Culture. Journal of Hubei University for Nationalities (Philosophy and Social Sciences), 31, 1-5.

Ziv, A. (1976). Facilitating Effects of Humor on Creativity. Journal of Educational Psychology, 68, 318-322. https://doi.apa.org/doi/10.1037/0022-0663.68.3.318 